\title{
Searches for supersymmetry in hadronic final states with the CMS detector
}

\author{
Christian Autermann* on behalf of the CMS collaboration \\ University of Hamburg \\ E-mail: christian.autermannedesy.de
}

CMS has searched for new physics in the fully hadronic final state. Because of the large branching fraction into quarks compared to charged leptons, analyses in this channel have generally large acceptances and therefore competitive sensitivities. The result of three independent and complementary analysis strategies are presented. The "Razor" analysis using $36 \mathrm{pb}^{-1}$ of luminosity and the $\alpha_{T}$ analysis, updated with $1.1 \mathrm{fb}^{-1}$, make use of different event-shape variables to supress the background. The third analysis searches in the missing transverse energy tail for an excess in $36 \mathrm{pb}^{-1}$ of data above the standard model background expectation modeled by data-driven methods. In the absence of any sign for new physics in the data exclusion contours in the constrained MSSM are derived and cross-section limits are interpreted in the framework of simplified models.

The 2011 Europhysics Conference on High Energy Physics-HEP 2011,

July 21-27, 2011

Grenoble, Rhône-Alpes France

\footnotetext{
* Speaker.
} 


\section{Introduction}

At CMS [1] several dedicated searches for supersymmetry (SUSY) are being pursued. Here, searches in the fully hadronic final state are discussed. These searches set today the tightest constraints on the supersymmetry parameter space, mainly because of the large branching fraction into quarks. The precise understanding of the dominant standard model (SM) background processes coming from QCD multi-jet, $Z \rightarrow v \bar{v}$, and $\bar{t} \bar{t}$ or vector-boson production where the lepton is not reconstructed is essential for all presented analyses. Three different search strategies are being followed: The "Razor" analysis aims at the reconstruction of the SUSY mass scale, while a complementary analysis makes use of an event-shape variable $\alpha_{T}$. A more inclusive search strategy is pursued in the "Jets and missing transverse energy (MET) analysis", with special emphasis on the data-driven modeling of the SM background.

\section{The jets and missing $E_{T}$ analysis}

To be as inclusive and model independent as possible, only jets and MET are required by this analysis [2]. The observed $H_{T}$ distribution in $36 \mathrm{pb}^{-1}$ of luminosity is compared to the SM simulation in Fig. 1(a). Great care was taken in order to model the SM background from the data. Events containing leptons are explicitly removed and are analyzed in other dedicated leptonanalyses. Three or more jets are required, the scalar sum of their transverse momenta defined as $H_{T}$ is used to trigger the events and is required to exceed $500 \mathrm{GeV}$. The QCD multi-jet background has no intrinsic MET, but jet resolution and jet mismeasurements due to various effects may lead to a large MET tail. This background is modeled using a multi-jet control sample, where the jets are rebalanced by maximizing a likelihood using the measured jet resolution, under the constraint

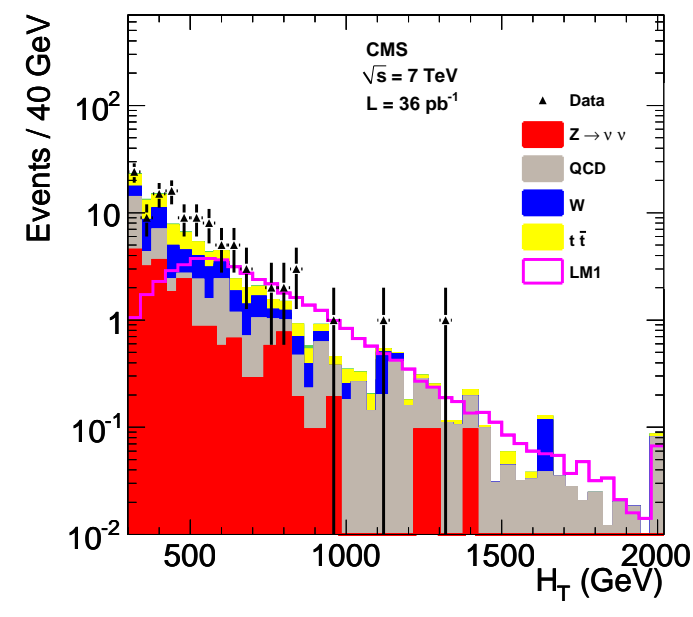

(a)

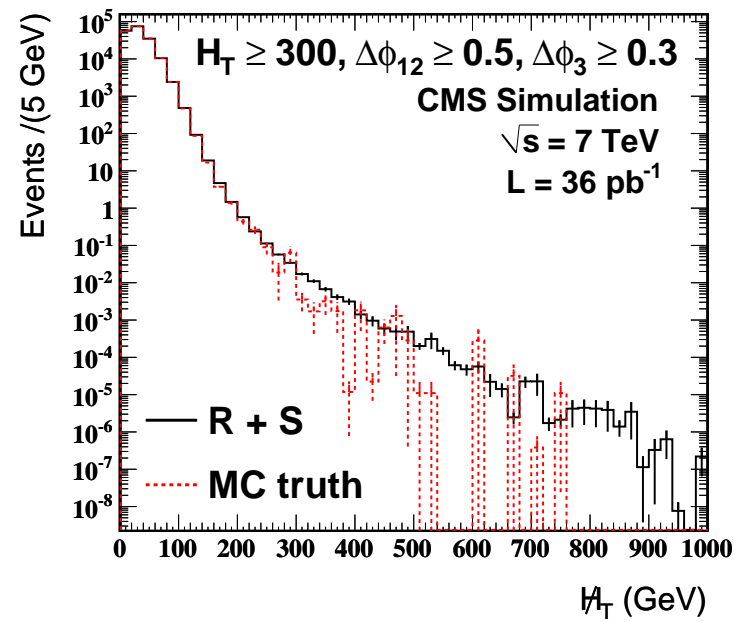

(b)

Figure 1: The observed data in $H_{T}$ compared to the expected SM background simulation (a) and the validation of the data-driven QCD multi-jet production in simulation with the direct expectation from simulation in $H_{\mathrm{T}}$, which is the negative vectorial sum of all energy clustered in jets (b). 
that the MET vanishes in all events. This rebalanced sample can be compared to QCD multi-jet events on generator level. Because of the rebalancing and the huge QCD cross-section the method is safe against signal contamination. In a second step, the jets of the rebalanced sample are smeared according to the jet resolution including non-Gaussian tails measured in $\gamma$-jet and di-jet data events. The method is shown in Fig. 1(b) to work for simulation. The $Z \rightarrow v \bar{v}$ background is modeled using $\gamma$-jet events, that share a similar kinematic if the boson transverse momentum is large compared to $m(Z)$. The uncertainty on the weighting factor $w\left(p_{T}^{\gamma}\right)$ has been calculated at NLO [3] and is smaller than $10 \%$. Background from $t \bar{t}$ and $W$ where the lepton is a hadronically decaying tau is modeled using a data control sample with one isolated muon. The muon is replaced by the hadronic response of a tau in the detector, using a response template constructed from simulation. If the leptons from $t \bar{t}$ or $W$ are not reconstructed, or not isolated, or not in the acceptance region, the lepton-veto fails to remove the event. This background is modeled using a similar isolated muon data sample, which is reweighed according to the muon or electron reconstruction and isolation efficiencies as measured on the Z-peak in data. The background from events with leptons out of the acceptance is taken from simulation. No excess over the data-driven background estimation is observed.

\section{The $\alpha_{\mathrm{T}}$ event-shape analysis}

The $\alpha_{T}$ analysis $[4,5]$ exploits an event-shape variable defined in a di-jet event as $\alpha_{T}=\frac{p_{T, j 2}}{M_{T}}$, with the transverse mass of the di-jet system $M_{T}=\sqrt{2 p_{T, j 1} p_{T, j 2} \cdot(1-\cos (\Delta \Phi))}$ and the transverse momentum of the next-to-leading jet $p_{T, j 2}$. The analysis requires two or more jets, in contrast to the previous discussed analysis, no explicit use of the MET variable is made and a luminosity of $1.1 \mathrm{fb}^{-1}$ is used. All jets are combined into an effective di-jet system, by maximizing $M_{T}$. While $\alpha_{T}$ has a long tail in events with real MET, events from QCD multi-jet production are well balanced in the transverse plane and therefore $\alpha_{T} \lesssim 0.5$ for QCD events. The observed data and the sharp drop of the QCD multi-jet background at $\alpha_{T} \approx 0.5$ is shown together with the remaining SM backgrounds in Fig. 2(a). A selection on $\alpha_{T}>0.55$ is applied, but no excess is observed in the data. In order to translate the measurement into limits on new physics, the different statistically independent bins of the $H_{T}$ distribution as shown in Fig. 2(b) are used as separate counting exper-

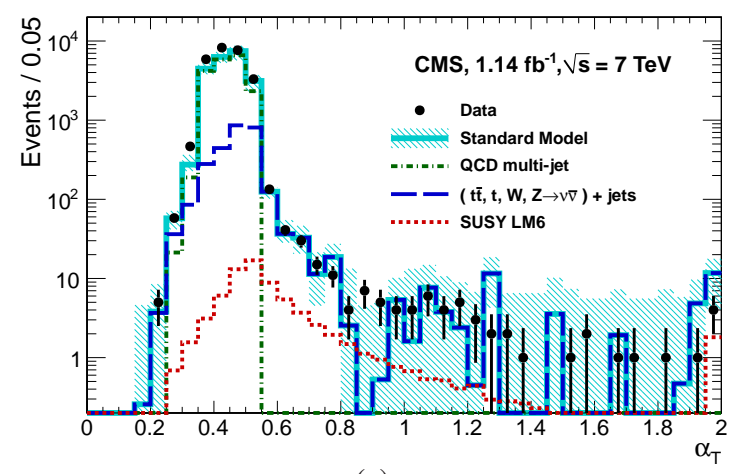

(a)

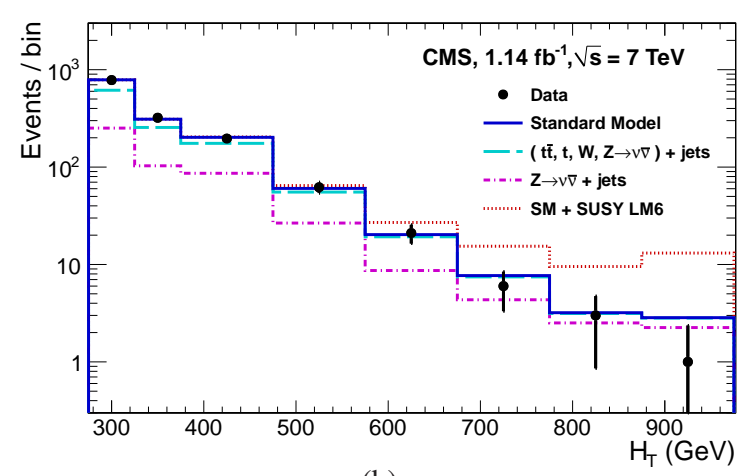

(b)

Figure 2: Observed and expected $\alpha_{T}$ (a) and the resulting $H_{T}$ distribution (b). 
iment channels with correlated systematic uncertainties. The normalization of the backgrounds is allowed to float; the shape of the remaining QCD background is described by a falling exponential but is statistically compatible with zero. The shapes of the remaining backgrounds are determined by data-driven methods in a similar way as discussed in the previous section.

\section{The "Razor" event-shape analysis}

The "Razor" analysis [6] has searched for two pair produced heavy particles like e.g. squarks or gluinos in $36 \mathrm{pb}^{-1}$ of data. All events are forced by hemisphere algorithms into two mega-jets $j_{1}$ and $j_{2}$, so that $M_{R}$ and $M_{R}^{T}$ are estimators of the involved mass scale $M_{\Delta}=\frac{M_{\tilde{q}}^{2}-M_{\tilde{\chi}_{1}^{0}}^{2}}{M_{\tilde{q}}}$ of the signal events, in the limit that the two squarks or gluinos are produced at threshold:

$$
M_{R}=2 \sqrt{\frac{\left(E_{j 1} \cdot p_{z, j 2}-E_{j 2} \cdot p_{z, j 1}\right)^{2}}{\left(p_{z, j 1}-p_{z, j 2}\right)^{2}-\left(E_{j 1}-E_{j 2}\right)^{2}}}, \quad M_{R}^{T}=\sqrt{\frac{E_{\mathrm{T}}\left(p_{T, j 1}+p_{T, j 2}\right)-\vec{E}_{\mathrm{T}}\left(\vec{p}_{T, j 1}+\vec{p}_{T, j 2}\right)}{2}} .
$$

$M_{R}$ is expected to peak at $M_{\Delta}$ for signal events, while for QCD multi-jet events $M_{R}$ is a measure of the center-of-mass energy of the relevant partonic subprocess $\sqrt{\hat{s}}$. This behavior is used in order to predict the amount of QCD background in the signal region at $M_{R}>500 \mathrm{GeV}$ and $R=\frac{M_{R}}{M_{R}^{T}}>0.5$ by extrapolation. $M_{R}$ falls as expected exponentially for QCD multi-jet production, as shown in Fig. 3(a) for different cuts on the massless ratio $R$. The remaining backgrounds with genuine MET are estimated from the data by explicitly requiring either a muon or an electron. After the application of the $R$ cut, the $M_{R}$ distribution for the lepton and the fully hadronic samples, where the lepton is not reconstructed, are sufficiently similar. This similarity is used to model the shape and the normalization of these backgrounds. The observed data and the total final data-driven background prediction is shown in Fig. 3(b).

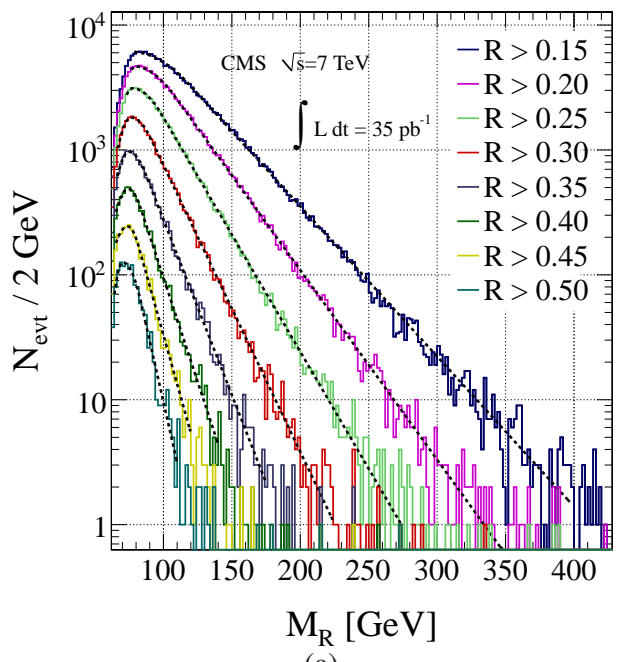

(a)

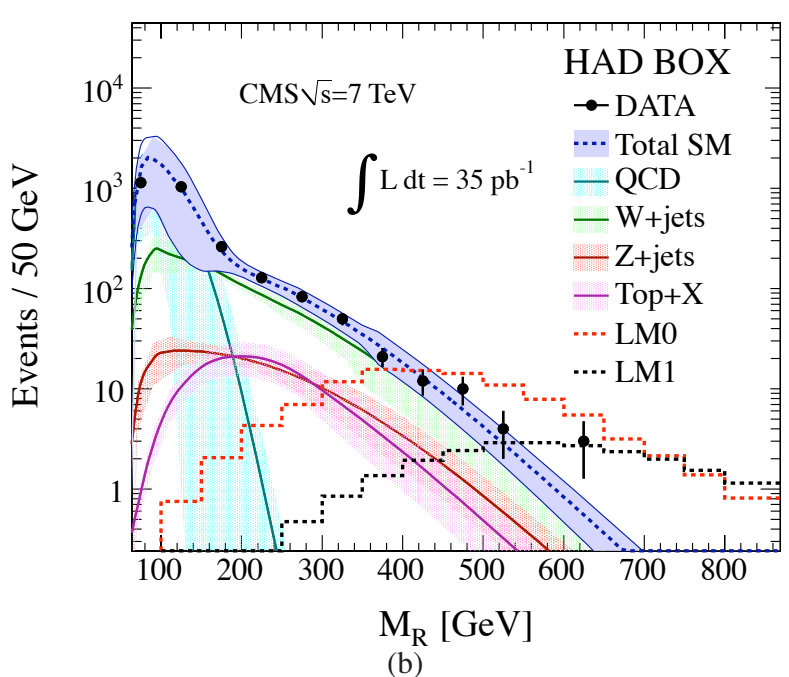

(b)

Figure 3: "Razor" variable $M_{R}$ for QCD data events and different cuts on $R$ (a), and the resulting prediction and observed data (b). 


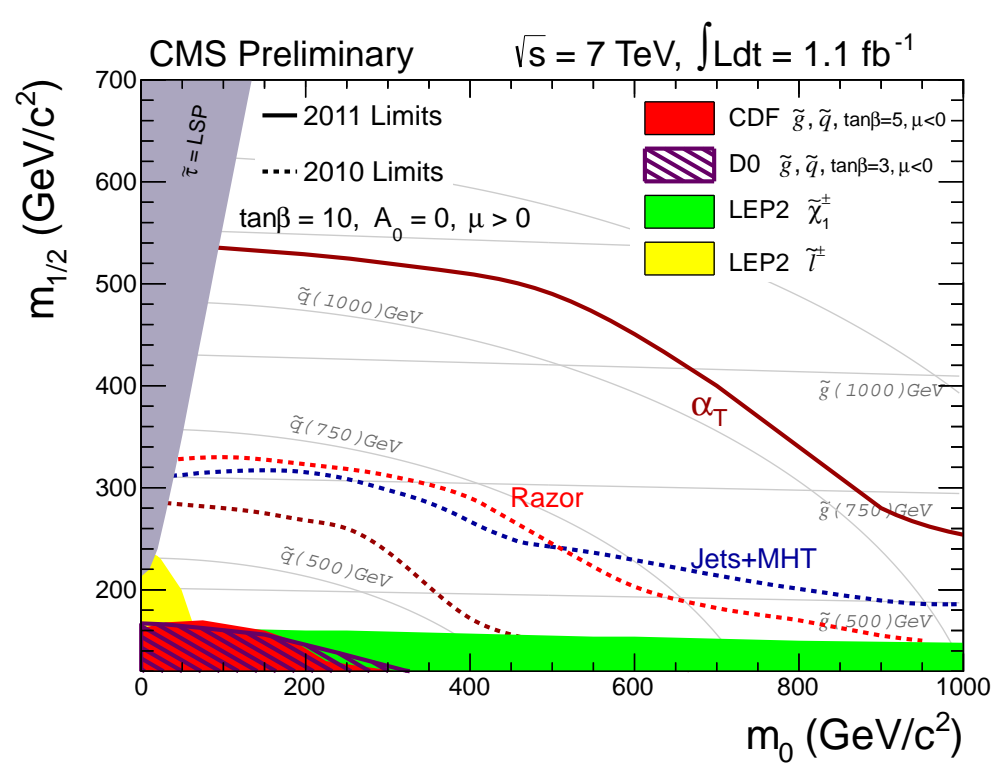

Figure 4: Summary of all analysis exclusion contours interpreted within the cMSSM framework.

\section{Results and Summary}

Three complementary searches for new physics in the fully hadronic final state have been performed. No excess in the data corresponding to an integrated luminosity of up to $1.1 \mathrm{fb}^{-1}$ has been observed. CLs limits have been calculated and interpreted in the CMSSM and the observed exclusion contours at 95\% confidence level are shown in Fig. 4, typically gluino $m(\tilde{g}) \leq 0.6 \mathrm{TeV}$ and squark masses $m(\tilde{g}) \leq 1.1 \mathrm{TeV}$ can be excluded. Cross-section limits in the context of the simplified model framework have been calculated and can be found at Ref. [7]. The analysis based on the MHT sensitive variable has been updated meanwhile with more than $1 \mathrm{fb}^{-1}$ of luminosity leading to comparable limits like the $\alpha_{T} 1 \mathrm{fb}^{-1}$ analysis [8].

\section{References}

[1] S. Chatrchyan et al. [ CMS Collaboration ], JINST 3 S08004 (2008).

[2] S. Chatrchyan et al. [ CMS Collaboration ], JHEP 1108:155 (2011), arXiv:1106.4503.

[3] Z. Bern et al., (2011) arXiv:1106:1423.

[4] S. Chatrchyan et al. [ CMS Collaboration ], (2011) arXiv:1109.2352.

[5] V. Khachatryan et al. [ CMS Collaboration ], Phys. Lett. B698 (2011) 196-218, arXiv:1101.1628.

[6] S. Chatrchyan et al. [ CMS Collaboration ], (2011) arXiv:1107.1279,

[7] The CMS Collaboration Public TWiki, "CMS Supersymmetry Physics Results", https://twiki.cern.ch/twiki/bin/view/CMSPublic/PhysicsResultsSUS.

[8] S. Chatrchyan et al. [ CMS Collaboration ], (2011) CMS-PAS-SUS-11-004. 